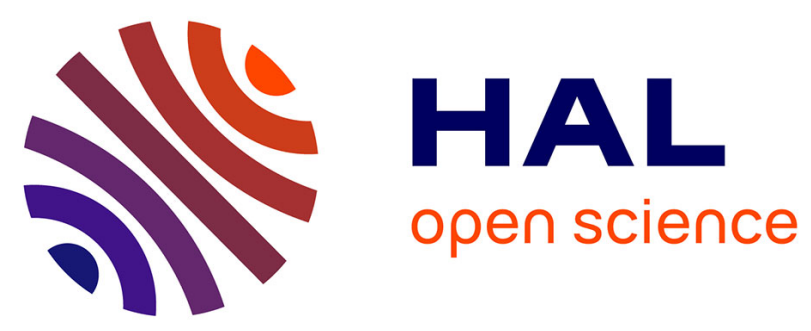

\title{
Investigating carbon materials nanostructure using image orientation statistics
}

Jean-Pierre da Costa, P. Weisbecker, B. Farbos, J.-M. Leyssale, G.L. Vignoles, C. Germain

\section{- To cite this version:}

Jean-Pierre da Costa, P. Weisbecker, B. Farbos, J.-M. Leyssale, G.L. Vignoles, et al.. Investigating carbon materials nanostructure using image orientation statistics. Carbon, 2015, 84, pp.14. 10.1016/j.carbon.2014.11.048 . hal-01120268

\section{HAL Id: hal-01120268 https://hal.science/hal-01120268}

Submitted on 25 Feb 2015

HAL is a multi-disciplinary open access archive for the deposit and dissemination of scientific research documents, whether they are published or not. The documents may come from teaching and research institutions in France or abroad, or from public or private research centers.
L'archive ouverte pluridisciplinaire HAL, est destinée au dépôt et à la diffusion de documents scientifiques de niveau recherche, publiés ou non, émanant des établissements d'enseignement et de recherche français ou étrangers, des laboratoires publics ou privés. 


\title{
Investigating carbon materials nanostructure using image orientation statistics
}

\author{
J.P. Da Costa ${ }^{a, b,{ }^{*},}$ P. Weisbeckerc, B. Farbos ${ }^{c, b}$, J.-M. Leyssalec, G. L. Vignoles ${ }^{d, c}$, C. \\ Germain $^{a, b}$ \\ ${ }^{a}$ Univ. Bordeaux, IMS UMR 5218, F-33400 Talence, France \\ ${ }^{\mathrm{b}}$ CNRS, IMS UMR 5218, F-33400 Talence, France \\ ${ }^{c}$ CNRS, LCTS UMR 5801, F-33600 Pessac, France \\ ${ }^{\mathrm{d}}$ Univ. Bordeaux, LCTS UMR 5801, F-33600 Pessac, France
}

\begin{abstract}
A new characterization method of the lattice fringe images of turbostratic carbons is proposed. This method is based on the computation of their orientation field without explicit detection of fringes. It allows meaningful insights into the material nanostructure and nanotexture at several scales, either qualitatively or quantitatively. The calculation of pairwise spatial statistics of the orientation field at short distance provides measurements of the coherence lengths along any direction, in particular along and orthogonally to the layers. These statistics also allow representing orientation coherence patterns typical of the observed nanostructure. At larger distances, the mean disorientation of the fringes is computed and information about the homogeneity of the sample is obtained. An experimental validation is carried out on various artificial images and an application to the characterization of four bulk turbostratic carbons is provided.
\end{abstract}

\section{Introduction}

Lattice fringe (LF) imaging using High Resolution Transmission Electron Microscopy (HRTEM) has been for more than two decades a choice technique for investigating and quantifying the nanostructure (i.e. the spatial arrangements of graphene layers) of carbonaceous materials such as soot [1], activated carbons [2], chars [3-5], pyrocarbons [6-9], or nuclear graphites [10-11].

Though meaningful and informative, the qualitative interpretation of LF images is subjective and time consuming. The need for quantitative analysis tools has rapidly lead to develop automated image analysis tools, as reported e.g. in refs. [3,12-14].

Whatever the type of carbon materials under study, most of published LF image analysis procedures are based on four main algorithmic steps, namely (i) filtering of the digitized Corresponding author. Tel: (33)5 40002634 E-mail : jean-pierre.dacosta@ ims-bordeaux.fr 
image, (ii) fringe extraction, (iii) morphological analysis of individual fringes, (iv) characterization of spatial arrangements of fringes.

Essentially, image filtering (i) aims at removing high frequency artifacts (mainly electronic noise) and low frequency variations (Bragg artifacts and sample thickness variations) that are not meaningful to describe material nanostructure and may hinder image analysis. Filtering is usually conducted in the Fourier domain by applying either ideal square filters $[2-3,13]$ or smooth Gaussian filters [15]. As mentioned by Toth and coworkers, the latter prevents harmonic ringing caused by the sharp changes in the transfer function and subsequent false fringe detections. In the case of more anisotropic materials such as rough or regenerated laminar pyrocarbons for instance [8], a combination of radial and angular filtering has also appeared to be a relevant technique to remove irrelevant details in LF images.

Typically, fringe detection (ii) starts with image binarization in order to discriminate fringe objects from background. Binarization is obtained by thresholding using either a fixed $[3,13]$ or an adaptive [15] threshold. Rouzaud and Clinard [13] and later Pré et al. [2] suggested the prior use of a top-hat transform to ease the thresholding process. Binarization is usually followed by fringe skeletonization and various post-processing operations in order to separate, reconnect or remove fringe chunks [2-3,12,14]. Another possible approach for fringe detection relies in the use of a sub-pixel level curve tracking algorithm initially proposed in [16] and later used in [17-18]. After image background variations removal, level curves are tracked on either side of the fringes around the mean intensity level until the fringe ends (zones with poor orientation coherence) are reached.

Morphological fringe analysis (iii) usually comprises statistics of individual fringe lengths and tortuosities [2-3,14,18], curvatures and orientations [2,15]. Shim et al. also proposed a measure of the apparent crystalline fraction [3].

Besides individual fringe features statistics, various authors addressed the description of mutual arrangements of fringes (iv) by measuring interlayer spacing histograms [2,13-14]. Toth et al. [15] also proposed to measure the interlayer spacing deviation parameter or the junction parameter (number of branch points per unit length). The identification of stacks of parallel fringes - assimilated to Basic Structural Units [19-20] - has also been addressed by several authors [2,13-15]. They established statistics for the stacking number $N$ and the coherence length $L_{a}$. Other valuable features that can be extracted from LF images are the 2D nematic and polar order parameters [3]. Computed from the orientations of the fringes within a given spatial window around any given location of the image, these order parameters 
quantify the organization of fringe orientations inside the window. In brief, the nematic order parameter gets high values where fringes are all nearly parallel while the polar parameter is high in the case of concentric fringes around the window center. Such parameters have later been used as saliency features to detect soot spherules in large images and drive an automated image analysis process [21].

Though yielding lots of relevant information about many material nanostructures, the bottleneck of this kind of approaches is the need for a fringe extraction algorithm that usually consists of numerous complex steps with various parameters, sometimes subjective and most of the time application specific. In recent works, some authors argue that valuable information can also be obtained without explicit fringe extraction. For instance, Toth et al. [22] proposed an original framework to compute statistics of interlayer spacing and fringe orientation based on the direct application of a Gabor filter bank on an LF image. In [23], the same authors presented an alternative approach for the computation of multiscale nematic and polar order parameters. In this approach, the orientation statistics were computed from the image orientation field estimated using steerable filters [24] without explicit identification of fringes. In former works, Germain et al. [25] also proposed a general framework for the multiscale evaluation of texture anisotropy using feature $I_{s o}$ which was used to discriminate between various raw and heat treated pyrocarbons.

In this paper, we address the characterization of spatial arrangements of fringes in turbostratic carbons through LF images and their orientation fields. The latter are computed by standard computationally efficient estimation techniques without explicit detection of fringes. We show that the visualization of the local orientation map and of the orientation coherences allows a more convenient representation of the nanotexture and of the defects. In addition, spatial statistics of the orientation field are used to gather information on the texture at several scales. While previous approaches based on the multiscale nematic order $[3,23]$ or on multiscale anisotropy [25] provide global estimations of the degree of anisotropy at one or several scales, the specificity of our work is to consider pairwise statistics, i.e. statistics of pairs of pixels. Using ad-hoc graphical representations, our approach allows monitoring how fringe orientation coherence vanishes between two sites according to distance and orientation, thus providing access to the grain shapes. The relevance of our approach is first assessed on artificial images showing grain tessellations before studying real material images. In particular, for highly textured materials as turbostratic carbons, we obtain coherence lengths at short distance, parallel and perpendicular to the layers, which are related to other coherence 
measures provided by X-ray diffraction (XRD). At larger distances, the mean angular deviation of the fringes is computed and information about the homogeneity of the sample is obtained.

The paper is organized as follows. The second section provides details about material samples, characterization techniques and image processing algorithms including orientation retrieval and spatial statistic computation. The third section deals with experimental validation on various artificial images and shows an application to the characterization of four types of bulk carbons. Finally, we will draw some conclusions and mention a few prospects for future work.

\section{Materials and methods}

\subsection{Material samples}

Four materials were chosen to evaluate this method: three pyrocarbons (PyCs) - namely the Rough Laminar PyC (RL), the Regenerated Laminar PyC (ReL) and the Smooth Laminar PyC (SL) - and a PAN-based carbon fiber $\left(\mathrm{C}_{\mathrm{Fiber}}\right)$. All materials are made of graphene layers stacked together with a turbostratic disorder between the layers. Differences between the materials lay in their texture (i.e. the preferential orientation of the layers), the curvature of their layers, and the shape and size distribution of their BSUs (Basic Structural Units).

The RL PyC [26] and the ReL PyC [7] are both high textured PyCs [6], the latter containing a higher amount of structural defects. The smooth laminar PyC (SL) is medium textured and more heterogeneous as it is made of a mixture of lamellae and pores of various sizes [27]. $\mathrm{C}_{\text {Fiber }}$ exhibits a distinct texture, isotropic when observed along the fiber axis and rather homogeneous with strongly folded layers, corresponding to the axial orientational order [3].

ReL, RL and SL PyCs were prepared by isothermal isobaric Chemical Vapor Infiltration (CVI) from respectively pure propane (ReL) and a methane/propane mixture (RL) precursors. The RL PyC was obtained by deposition on the surface of a fused quartz tube filled at both ends by a carbon preform and placed in the CVI reactor [28] while the SL PyC was obtained in the same conditions, but with the fused quartz tube left open. For the ReL PyC, a silica woven fabric was infiltrated using pure propane with the following processing parameters: total pressure $p=5 \mathrm{kPa}$, temperature $T=1050^{\circ} \mathrm{C}$, residence time $t_{r}=3$ seconds and 10 hours infiltration duration [29]. The carbon fiber is a commercial ex-PAN carbon fiber [30]. 


\subsection{Material characterization}

\subsubsection{HRTEM imaging}

Before being studied by TEM, bulk samples were embedded in epoxy glue, mechanically polished down to $100 \mu \mathrm{m}$ using a $15 \mu \mathrm{m}$ diamond plate and then ion thinned in order to achieve electron transparency using a JEOL Ion Slicer (EM-09100IS) [31]. The initial incidence angle was set to $0.5^{\circ}$ with an energy of $5.5 \mathrm{keV}$ for the $\mathrm{Ar}^{+}$ion beam; then, the angle was increased to $2.5^{\circ}$ and the energy progressively reduced to $4 \mathrm{keV}$ and eventually 2 $\mathrm{keV}$ in order to reduce the irradiation damage to the carbon structure.

High-resolution transmission electron microscopy (HRTEM) images were acquired on a Philips CM30ST TEM (300 kV - LaB $)$. All images were obtained near Scherzer defocus $(-57 \mathrm{~nm})$ with an objective aperture of $4 \mathrm{~nm}^{-1}$ allowing to select only the 002 lattice fringes. Near Scherzer defocus the contrast transfer function (CTF) of the transmission electron microscope (TEM) is nearly flat for an extended region of spatial frequencies (roughly from 2 to $4.2 \mathrm{~nm}^{-1}$ ), including the frequency domain corresponding to the 002 lattice fringes $\left(2.9 \mathrm{~nm}^{-1}\right)$. In these conditions the atoms appear in black in the LF image which can be seen, as a first approximation, as an inverted image of the projected potential (projection along the TEM optical axis through the thin foil thickness). It is well known that graphitic structures are prone to amorphization under a $300 \mathrm{kV}$ electron beam [32], hence the acquisition time was reduced to 10 seconds for each zone in order to reduce the irradiation dose as much as possible.

\subsubsection{X-ray diffraction}

RL and ReL PyCs were analyzed by powder diffraction using a Bruker D8 Advance diffractometer $\left(\lambda \mathrm{K}_{\alpha} \mathrm{Cu}\right)$ in order to determine the coherence lengths along the $c$-axis $L_{c}$ and in the plane $L_{a}$. Powders were filled into a zero background sample holder and the data were acquired on a $2 \theta$ range of $10-74^{\circ}$. The whole patterns were fitted using the CarbonXS software [33] which accounts for the strong overlap between the 10 band and the 004 peak. The SRM1976 (corundum) calibration standard was used to check the alignment of the diffractometer and to determine the instrumental resolution which is smaller than $0.07^{\circ 2 \theta}$ while carbon peaks are larger than $1.6^{\circ 2 \theta}$. Thus the instrumental resolution was not taken into account as it is negligible comparing to the pyrocarbons peaks widths. 


\subsection{Image processing}

LF images belong to the category of what is called textured images. Texture is a keystone of many image analysis tasks including classification, segmentation or characterization. In order to extract texture descriptors, statistical approaches based on second and higher order statistics have been widely reported in literature. For instance, methods based on the autocorrelation and on co-occurrence matrices [36] or on gray level difference histograms [37-38] are simple and efficient to extract texture descriptors. These methods rely only on the distribution of pixel intensity values. Several authors [39-41] pointed out that methods based on gray level statistics are not suited to the description of structured textures. Indeed, textures arising from an arrangement of local patterns cannot be described only in terms of gray level variations. Descriptors related to the spatial arrangement of texture patterns are needed. Pattern orientations have been recognized by several authors as important features for machine vision systems [25,38-43]. Although the extraction and the regularization of pixel orientations have been widely addressed, e.g. in [42,44-46], very few studies have focused on the use of spatial statistics of the orientation field. In their pioneering works, Davis et al. [39] presented a generalization of co-occurrence matrices accounting for pattern orientations. They defined the generalized co-occurrence matrix as a multidimensional array where each element is a count of the number of occurrences of a given spatial configuration of pixels. This configuration includes the relative position of the pixels and any kind of features characterizing the pixels. The generalized co-occurrences were applied to the magnitudes and orientations of the gradient vectors, computed on object boundaries. Later, Kovalev et al. [40-41] provided implementations of multidimensional co-occurrence matrices. They proposed either to combine gray level and orientation statistics [40] or to base the co-occurrences on the orientation field only [41]. Their approach, applied to a small set of spatial relations, proved to be efficient for the discrimination of specific textures with local anisotropies.

In the following we propose an original framework for the second order statistical analysis of the orientation field. Based on a rotationally invariant implementation of orientation spatial differences, it specifically addresses the case of anisotropic textures such as those appearing in HRTEM LF images.

\subsubsection{Image filtering}

HRTEM image filtering is carried out according to the radial-angular filter used, though not detailed, in [8-9,47]. The filter performs in the Fourier domain and is defined by its band-pass transfer function: 


$$
\mathrm{H}(\rho, \theta)=\mathrm{H}_{\text {radial }}(\rho) \mathrm{H}_{\text {angular }}(\theta)
$$

with

$$
\mathrm{H}_{\text {radial }}(\rho)=\frac{1}{\sigma_{\rho} \sqrt{2 \pi}} e^{-\frac{\left(\rho-\rho_{0}\right)^{2}}{2 \sigma_{\rho}^{2}}} \text { and } \mathrm{H}_{\text {angular }}(\theta)=\frac{1}{\sigma_{\theta} \sqrt{2 \pi}} e^{-\frac{\left(\theta-\theta_{0}\right)^{2}}{2 \sigma_{\theta}^{2}}}
$$

$(\rho, \theta)$ are the polar frequency coordinates in the Fourier domain. $\rho_{0}=\frac{1}{d_{002}} \approx 2.9 \mathrm{~nm}^{-1}$ corresponds with the interlayer spacing. $\sigma_{\rho}=\alpha \rho_{0}$ is defined from $\rho_{0}$ with $0<\alpha<1$. $\mathrm{H}_{\text {radial }}$ performs a Gaussian filtering around $\rho=\rho_{0}$, reducing high and low frequency artifacts while preserving $d_{002}$ fringes whatever their orientation. The second term $\mathrm{H}_{\text {angular }}$ aims at preserving patterns which are oriented around a reference orientation $\theta_{0}$ that fits the preferential orientation in the image. $\theta_{0}$ is found by looking for the largest magnitude peak in the Fourier transform modulus. Filtering is done using a Gaussian function centered on $\theta_{0}$ with a standard deviation of $\sigma_{\theta}$. The typical values $\alpha=0.5$ and $\sigma_{\theta}=50^{\circ}$ have proved to be a good compromise between artifact removal and structure preservation, (see e.g. [8-9]), for anisotropic PyCs such as the RL and ReL PyCs. For poorly anisotropic materials such as the SL PyC and $\mathrm{C}_{\text {Fiber, }}$, angular filtering is deactivated in order to prevent the apparition of unwanted artificial structures in the filtered image. Naturally, though guided by experience, the determination of $\alpha$ and $\sigma_{\theta}$ remains arbitrary and is subject to the appreciation of the experimenter. Nevertheless, when kept within acceptable ranges leading to eye-friendly images, the choice of values is not critical provided that the values are kept constant when comparing various material images together. Indeed, variations in the filter strength or in the filter nature do not hinder the calculation of orientations statistics contrary to fringe detection algorithms that directly depend on image grey level variations. A comparison of various filtering approaches is provided in the supplementary material, showing their effect on the structural characteristics extracted from the orientation field. It is shown that in the absence of filtering - or equivalently in the presence of noise - the orientation maps can still be estimated and their statistical analysis still provides information about orientation spatial coherence. However, the presence of high frequency noise may lead to slightly underestimate the material anisotropy, which can be easily avoided by filtering.

\subsubsection{Orientations: definition, estimation and representation}

The second step of the LF image processing process is the estimation of the orientation field. Various definitions of orientation are available in image processing literature. For instance, the linearly symmetric model [44], the ilD case [48] or the mono-directional model [46] 
provide definitions we can refer to. They all consider that a unique orientation is available at any location, i.e. it is the orientation of the underlying texture pattern.

In the case of locally unique orientations, a standard approach to extract local orientations in an image is the local structure tensor (LST) [42], based itself on the image intensity gradient. Let $I$ be the image magnitude and $\Omega \subset \mathbb{N}^{2}$ the image pixel grid. The image intensity gradient at location $\mathbf{u} \in \Omega$ is defined by:

$$
\boldsymbol{\nabla} I(\mathbf{u})=\left[\begin{array}{l}
I_{x}(\mathbf{u}) \\
I_{y}(\mathbf{u})
\end{array}\right]=\left[\begin{array}{c}
\frac{\partial I(\mathbf{u})}{\partial x} \\
\frac{\partial I(\mathbf{u})}{\partial y}
\end{array}\right]
$$

where $I_{x}$ and $I_{y}$ are the partial derivatives of the image. Gradient estimation is usually done using convolution masks in the spatial domain such as local derivative operators (e.g. [45]) or scalable gradient filters as Deriche's filter or Gaussian derivatives.

The LST at location $\mathbf{u}$ is the local covariance of the gradient field:

$$
\mathbf{T}(\mathbf{u})=\left[\begin{array}{cc}
\mathrm{T}_{\mathrm{xx}}(\mathbf{u}) & \mathrm{T}_{\mathrm{xy}}(\mathbf{u}) \\
\mathrm{T}_{\mathrm{xy}}(\mathbf{u}) & \mathrm{T}_{\mathrm{yy}}(\mathbf{u})
\end{array}\right]=\left\langle\boldsymbol{\nabla}(\mathbf{u}) \boldsymbol{\nabla}^{\dagger}(\mathbf{u})\right\rangle_{W_{\mathbf{u}}}=\left[\begin{array}{cc}
\left\langle I_{x}{ }^{2}(\mathbf{u})\right\rangle & \left\langle I_{x}(\mathbf{u}) I_{y}(\mathbf{u})\right\rangle \\
\left\langle I_{x}(\mathbf{u}) I_{y}(\mathbf{u})\right\rangle & \left\langle I_{y}{ }^{2}(\mathbf{u})\right\rangle
\end{array}\right]
$$

where $\dagger$ denotes matrix transpose and $\langle\cdot\rangle_{W_{\mathbf{u}}}$ denotes local average over a window $W_{\mathbf{u}}$ centered on $\mathbf{u}$. Either rectangular or Gaussian averaging windows can be considered, the latter having a more isotropic behavior.

The orientation $\theta(\mathbf{u})$ of the eigenvectors associated with the second eigenvalue of the LST indicates the local pattern direction, that is the orientation of minimum variability. This orientation estimate may be accompanied by a measure of confidence. A normalized confidence index is defined as $\eta(\mathbf{u})=\left(\lambda_{+}(\mathbf{u})-\lambda_{-}(\mathbf{u})\right) /\left(\lambda_{+}(\mathbf{u})+\lambda_{-}(\mathbf{u})\right)$ where $\lambda_{+}>\lambda_{-}$ are the eigenvalues of $\mathbf{T}(\mathbf{u})$ and $\eta(\mathbf{u}) \in[0,1]$. For each location $\mathbf{u}, \eta(\mathbf{u})$ is a local measure of collinearity between gradient vectors. This is a local measure of anisotropy. On lattice fringe images, $\eta(\mathbf{u})$ is close to 1 on straight fringes whereas it is close to 0 on fringe endings, on fringe dislocations, on amorphous areas or on overlapped layers.

The size of the averaging window $W_{\mathbf{u}}$ for the LST computation must be tuned according to the expected scale of the orientation estimation. In other words it must fit the size of the patterns of interest. In particular, in the case of lattice fringe images, the size of the averaging window must be tuned to the width of a fringe, the $d_{002}$ distance. Regarding the gradient estimation prior to the LST computation, any operator based on convolution filters may be 
chosen provided that their spatial extent is small compared to the size of the LST averaging window. For instance, $3 \times 3$ convolutive filters such as Sobel's, Prewitt's or GOP3 [45] are eligible.

Many other orientation estimation methods exist including those based on the use of filter banks such as the IRON operators [46] or the steerable filters [24] used in [23] for anisotropy analysis on soot images. The main advantage of filter bank approaches is their ability to detect and discriminate between several orientations simultaneously present on a site [46] as it may occur in LF images of soot, coal chars or activated carbons images for instance. In most parts of turbostratic carbon images, fringes can be considered as elongated patterns with a unique orientation and without pattern superimpositions. In this case, the use of the structure tensor, much more efficient computationally speaking and far simpler to implement, is more relevant.

Let us note that pattern orientations can be handled as axial data, i.e. $\pi$-periodic circular data [49]. Indeed two opposite angles, $\theta_{0}$ and $\theta_{1}=\theta_{0}+\pi$, relate to the same pattern orientation. Consequently, orientations $\theta(\mathbf{u})$ retrieved from the LST can be restricted to an interval of width $\pi$ such as $[0, \pi[$.

The couple composed of $\theta(\mathbf{u})$ and $\eta(\mathbf{u})$ (also noted $\theta_{\mathbf{u}}$ and $\eta_{\mathbf{u}}$ ), can be represented as a vector field $\mathbf{V}_{\mathbf{u}}=\left(\eta_{\mathbf{u}} \cos \theta_{\mathbf{u}}, \eta_{\mathbf{u}} \sin \theta_{\mathbf{u}}\right)^{\dagger}$ from which it is possible to extract spatial pairwise statistics as we will see below.

\subsubsection{Spatial statistics of orientations}

An intuitive way to measure the spatial dependence of orientations is to compute spatial orientation differences. In order to deal with the axial nature of orientation data, some of us [50] proposed to measure the difference between two orientations $\left(\theta_{1}, \theta_{2}\right) \in[0, \pi[\times[0, \pi[$ by using the function $\Delta\left(\theta_{1}, \theta_{2}\right)=\min \left(\left|\theta_{1}-\theta_{2}\right|, \pi-\left|\theta_{1}-\theta_{2}\right|\right)$. Based on $\Delta$, various secondorder features were proposed to describe orientation dependencies in texture, among which the $M O D$ feature defined by:

$$
\operatorname{MOD}(\mathbf{w})=\left\langle\eta_{\mathbf{u}} \eta_{\mathbf{u}+\mathbf{w}} \Delta\left(\theta_{\mathbf{u}}, \theta_{\mathbf{u}+\mathbf{w}}\right)\right\rangle_{\Omega}
$$

where $\langle\cdot\rangle_{\Omega}$ denotes spatial averaging over the whole image domain $\Omega . M O D$ can be considered as a weighted measure of similarity between two orientations separated by spatial lag $\mathbf{w} \in \mathbb{R}$.

We propose here to use a new rotation invariant formulation. Let $(\rho, \psi)$ be the polar representation of lag $\mathbf{w}$. The rotation invariant formulation consists in considering the polar 
shift $(\rho, \psi)$ not in the image grid coordinate system but in a local system defined by the local orientation:

$$
\begin{gathered}
\operatorname{RIMOD}(\rho, \psi)=\left\langle\eta_{\mathbf{u}} \eta_{\mathbf{u}+\boldsymbol{\delta}} \Delta\left(\theta_{\mathbf{u}}, \theta_{\mathbf{u}+\boldsymbol{\delta}}\right)\right\rangle_{\Omega} \\
\text { with } \boldsymbol{\delta}=\left(\rho \cos \left(\theta_{\mathbf{u}}+\psi\right), \rho \sin \left(\theta_{\mathbf{u}}+\psi\right)\right) .
\end{gathered}
$$

This feature allows measuring orientation dependencies when moving in a specific direction regarding the local structure orientation as shown in figure 1.

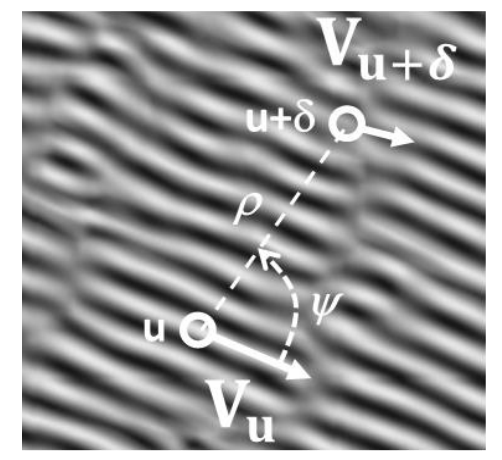

Figure 1. Polar representation of the spatial lag $(\rho, \psi)$ used in the case of the rotation invariant formulation RIMOD.

Note that in the case of non homogeneous lattice fringe images, with amorphous areas or regions with overlapped fringes, the computation of the features could be restricted to a region of interest (ROI) instead of being carried out over the entire image domain $\Omega$. Such an ROI could be delineated by hand or by a coarse segmentation process.

Various relevant graphical representations can be used to plot the RIMOD statistic as a function of the two variables $\rho$ and $\psi$. The ones provided in figures 3, 4, 5 and 9 plot $\operatorname{RIMOD}\left(\rho, 0^{\circ}\right)$ and $\operatorname{RIMOD}\left(\rho, 90^{\circ}\right)$ as a function of $\rho$. These representations allow to analyze how orientation coherence vanishes with distance and also to differentiate between the longitudinal (i.e. along the fringes) and transverse (i.e. across fringes) losses of coherence. A second possibility, illustrated in figure 10, consists of a radar representation (in polar coordinates) of the distance $\rho$ at which, for each angle $\psi$, the RIMOD feature reaches a given value $\varphi$. This second type of plot is very useful in identifying a sort of average coherence pattern, typical of the observed material structure. Though not used in this paper, other possible representations of such bivariate spatial statistics have been proposed in literature as for instance the feature-based interaction maps which are intensity coded representations of a spatial feature as a function of the spacing vector [51]. 
Regarding computational aspects, the approach proceeds in three major steps; $(i)$ image filtering, (ii) calculation of the orientation and coherence maps and (iii) calculation of the pairwise statistics. For an image of size $N=N_{x} \times N_{y}$, step $(i)$ is performed very efficiently with a computational complexity of $O(N \log N)$ in the Fourier domain. The complexity of step (ii) is $O\left(N \times W_{x} \times W_{y}\right)$, where $W_{x}$ and $W_{y}$ are the dimensions of the tensor averaging window. It is linear in $N$, allowing very low computational needs. Steps (i) and (ii) can thus be performed in a few seconds in any personal computer. In comparison, step (iii) is more time consuming as it involve a computational complexity of $O\left(N \times N_{\psi} \times N_{\rho}\right)$ where $N_{\psi}$ and $N_{\rho}$ are the number of angles and distances to be investigated in the pairwise statistics.

Typically, for an image of size $76 \mathrm{~nm} \times 76 \mathrm{~nm}$, acquired at a resolution $r=0.037 \mathrm{~nm} /$ pixel, the computation of $\operatorname{RIMOD}(\rho, \psi)$ for $N_{\psi}=2$ angles $\left(\psi=0^{\circ}\right.$ and $\left.90^{\circ}\right)$ and $N_{\rho}=67$ distances (between 0 and $5 \mathrm{~nm}$, every $0.074 \mathrm{~nm}$ ) takes around 10 minutes on a $2.5 \mathrm{GHz}$ processor, without parallelization.

\section{Results and discussion}

\subsection{Validation of the method on artificial images}

To illustrate and evaluate which kind of quantitative information can be provided by orientation statistics, artificial lattice fringe images were generated. The latter are Voronoi tessellations filled with 002 fringes spaced by $0.34 \mathrm{~nm}$. A tessellation is obtained by first generating a given number of seeds randomly in the image and, second, by assigning each pixel to the closest seed, in the sense of the Euclidean distance. The shapes of the Voronoi domains are not explicitly defined but are the result of the assignment process. Frontiers between neighboring domains are linear segments. Note that the shapes of the domains can be easily modified by weighting horizontal and vertical coordinates differently in the distance definition. For instance, if the horizontal coordinate differences are weighted twice as much as the vertical ones, the tessellation process results in rather vertical domains with a shape anisotropy factor of two (here a Y/X growth ratio of two).

Domain orientations are realizations either of $(i)$ a wrapped normal random variable [49] with zero mean (horizontal fringes) and with a given standard deviation, or (ii) a uniform $\left[0,180^{\circ}\right]$ random variable. Examples of such images are shown in figure 2. The full images have a size of $2048 \times 2048$ pixels and contain 2552 domains; the resolution was fixed to $0.037 \mathrm{~nm} /$ pixel i.e. the resolution of the experimental digitized HRTEM images used in this work. Thus the 
average grain area is $S_{g}=2.25 \mathrm{~nm}^{2}$ and the average grain dimension $D=\sqrt{S_{q}}=1.5 \mathrm{~nm}$. The two images shown in figure 2 only differ by the orientations of their fringes. The image in fig. 2 a corresponds to a highly textured case while fig. $2 \mathrm{~d}$ corresponds to an isotropic case. Images have been filtered with the radial frequency filter with $\rho_{0}=\frac{1}{d_{002}} \approx 2.9 \mathrm{~nm}^{-1}$ in order to smooth grain boundaries. Both orientation maps (Fig $2 \mathrm{~b}$ and $2 \mathrm{e}$ ) show that individual grain orientations are clearly identified while orientation confidence maps (Fig 2c and 2f) highlight the regions of disorder mainly located at grain boundaries.
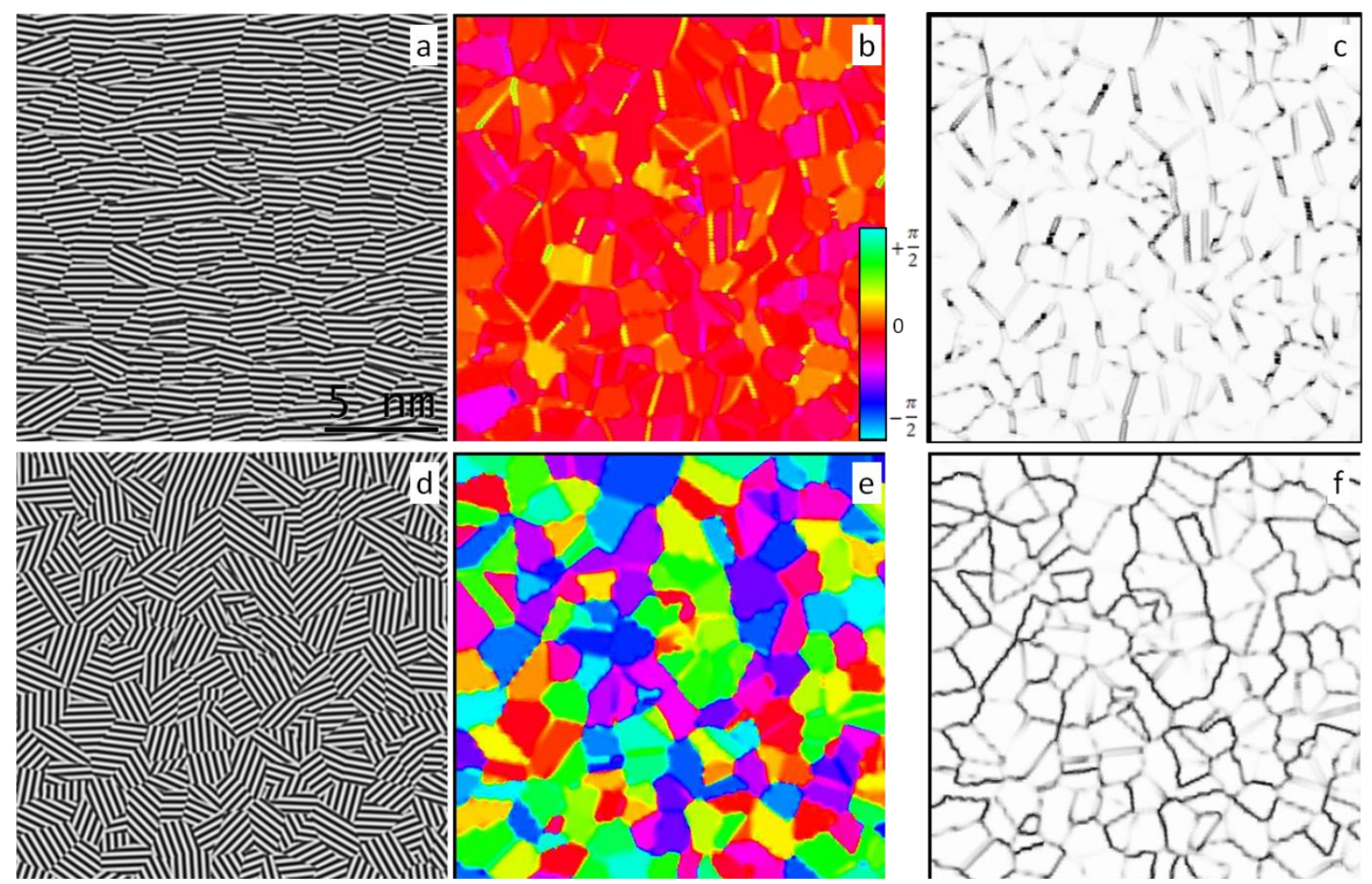

Figure 2: zoom of two artificial lattice fringe images (a,d) and corresponding local orientation $(b, e)$ and orientation confidences (c,f). The parameters used for the simulation are: images of $2048 \times 2048$ pixels, 2552 grains, standard deviation of grain orientations $\sigma=9^{\circ}$ for picture (a) and uniform orientation distribution for picture (d). Both images were preprocessed with a band-pass radial filter. Resolution is $0.037 \mathrm{~nm} / \mathrm{pixel}, d_{002}=0.34 \mathrm{~nm}$.

Plots of feature $\operatorname{RIMOD}(\rho, \psi)$ for both images, given at fig. 3, show the orientation difference as a function of distance for $\psi=0^{\circ}$ (along the fringes) and $\psi=90^{\circ}$ (orthogonally to the fringes). Both diagrams have a similar shape: a quick loss of orientation at short distance and 
a plateau reached at roughly $2.5 \mathrm{~nm}$. However the maximum value of orientation difference is around $12^{\circ}$ in the textured sample and $45^{\circ}$ in the isotropic sample. The loss of orientation at short distance is due to the interaction between neighboring grains and the plateau corresponds to the mean disorientation between grains. This plateau is reached for distances larger than the largest domains, thus giving domain size information. In order to obtain coherence lengths that can be accurately determined we have selected two parameters corresponding to the distance at which $90 \%$ of the value of the plateau is reached for the diagrams at $0^{\circ}$ and $90^{\circ}$. These parameters $L_{a M O D}$ and $L_{C M O D}$ are good estimates of the domain sizes as it is shown in table 1. It is also shown that the match between the domain size and $L_{C M O D}$ and $L_{A M O D}$ is good for domain sizes ranging from 0.5 to $3 \mathrm{~nm}$. The value of the plateau, $\beta_{M O D}$ is a quantification of the mean twist disorientation and provides information similar to the orientation angle $\left(\mathrm{OA}^{\circ}\right)$ obtained by electron diffraction but at a lower scale. From table 1 it can be see that $\beta_{M O D}$ increases when the domain size decreases (standard deviation of orientation $\sigma=9^{\circ}$ for all images); this is due to the higher amount of grain boundaries whose orientation is usually more difficult to determine. The ratio $L_{C M O D} / L_{a M O D}$ appears to be slightly larger than unity, suggesting a better orientation coherence orthogonally to the fringes. It can be attributed to the phase shift of the fringes of two neighboring grains having similar orientation; this leads to sharp transitions at the grain boundaries in the fringe direction while transitions are smoother in the direction perpendicular to the fringes.
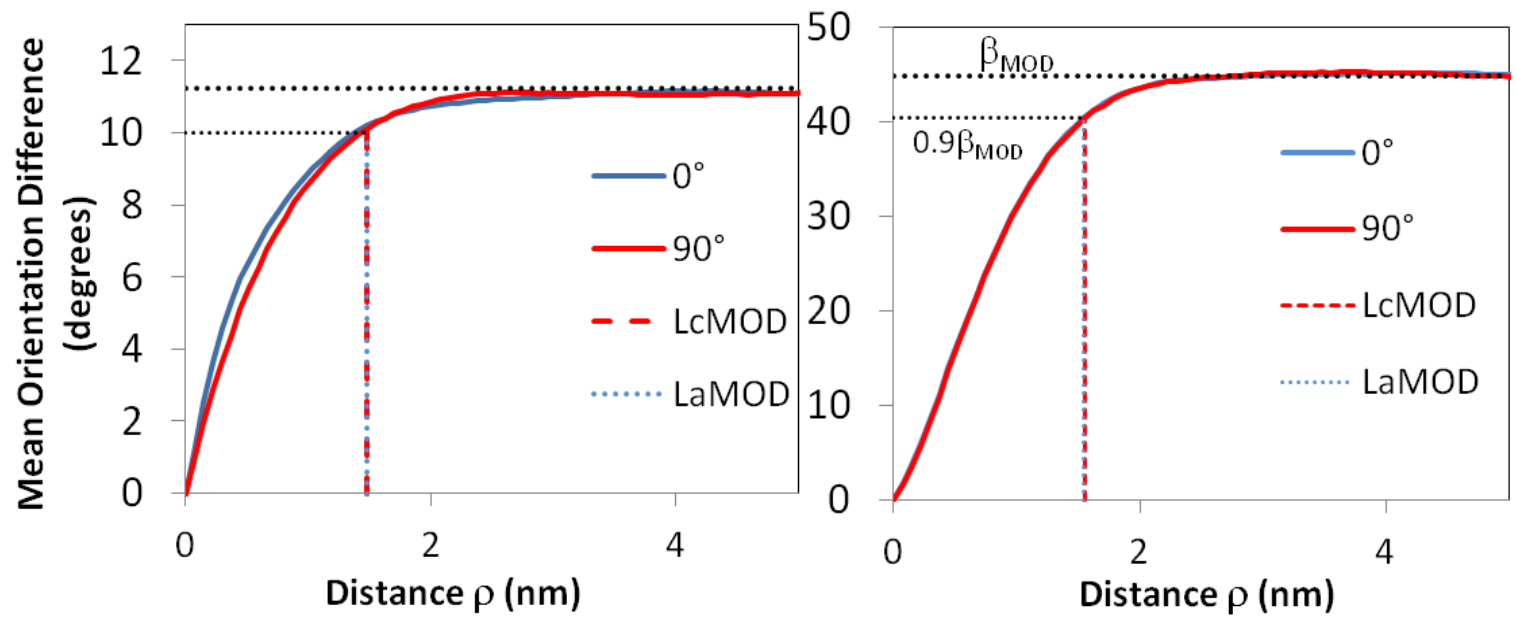

Figure 3: Plots of $\operatorname{RIMOD}\left(\rho, 0^{\circ}\right)$ and $\operatorname{RIMOD}\left(\rho, 90^{\circ}\right)$ for the two images in figure 2, high textured (left) and isotropic sample (right), dotted lines correspond to the parameters $\beta_{\mathrm{MOD}}$, $L_{C M O D}$ and $L_{a M O D}$. 


\begin{tabular}{|c|c|c|c|c|c|c|c|}
\hline $\begin{array}{c}\text { Image Size } \\
\text { (pixels) }\end{array}$ & $\mathrm{N}$ & $\mathrm{D}(\mathrm{nm})$ & $\sigma^{\circ}$ & $L_{c M O D}(\mathrm{~nm})$ & $L_{a M O D}(\mathrm{~nm})$ & $\frac{L_{c M O D}}{L_{a M O D}}$ & $\beta_{M O D}{ }^{\circ}$ \\
\hline $1024 \times 1024$ & 5742 & 0.50 & 9.0 & 0.54 & 0.47 & 1.14 & 13.2 \\
\hline $1024 \times 1024$ & 1346 & 1.03 & 9.0 & 1.06 & 0.97 & 1.09 & 11.7 \\
\hline $2048 \times 2048$ & 2552 & 1.50 & 9.0 & 1.48 & 1.44 & 1.03 & 11.2 \\
\hline $2048 \times 2048$ & 1436 & 2.00 & 9.0 & 2.06 & 2.05 & 1.01 & 11.0 \\
\hline $2048 \times 2048$ & 638 & 3.00 & 9.0 & 2.92 & 2.85 & 1.03 & 10.9 \\
\hline $2048 \times 2048$ & 2552 & 1.50 & isotropic & 1.55 & 1.54 & 1.01 & 44.9 \\
\hline
\end{tabular}

Table 1: determination of $L_{C M O D}, L_{a M O D}$ and $\beta_{M O D}$ from various artificial lattice fringe images. $n$ is the number of domains in the image and $D$ is the mean domain size.

Experimental images usually exhibit domains with an anisotropic shape. In order to study the efficiency of our method with such a microstructure, we have generated artificial images with columnar grains and with fringes preferentially oriented perpendicular to the columns. Figure 4 shows the corresponding RIMOD diagram for columnar grains, it appears that curves at $0^{\circ}$ and $90^{\circ}$ are clearly different, leading to a ratio $L_{c M O D} / L_{a M O D}$ of 1.8 close to the shape anisotropy of 2 used to synthesize the image. Therefore $L_{C M O D}$ and $L_{a M O D}$ give information about domain sizes perpendicular and parallel to the layers.
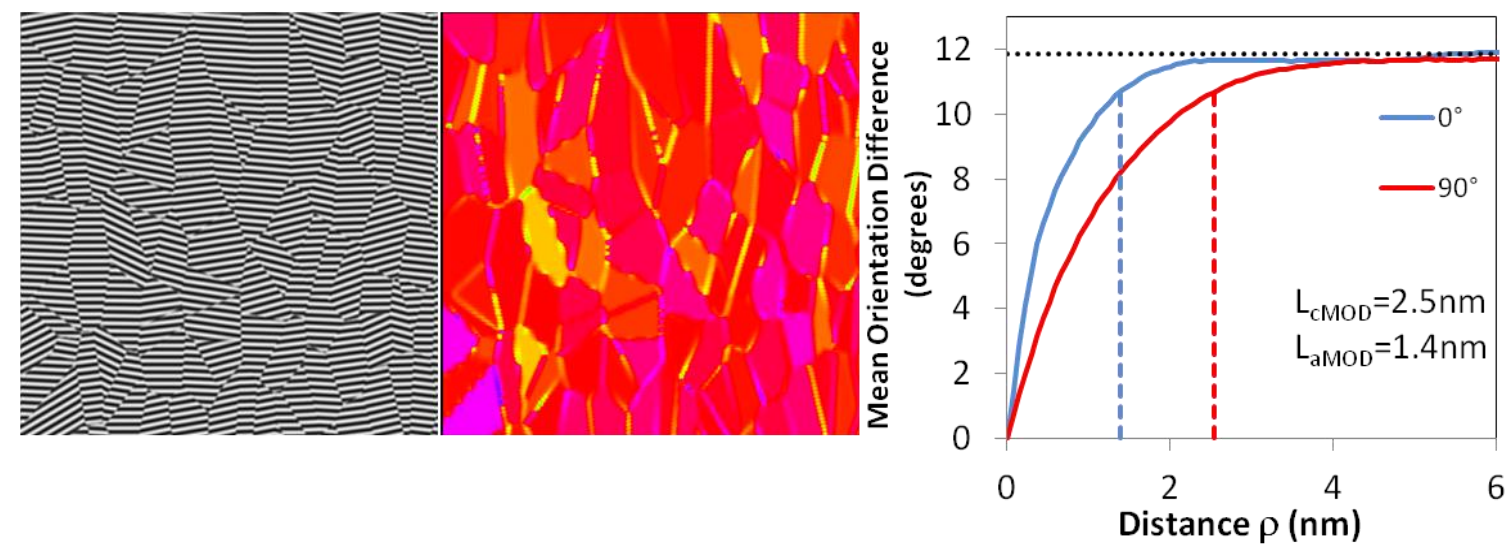

Figure 4: Mean orientation difference diagram corresponding to an image with columnar domains and zoom of the corresponding image and of the local orientation map. The size of the full image used for orientation statistics computing is $2048 \times 2048$ pixels $\left(5742 \mathrm{~nm}^{2}\right)$. It 
contains 1436 domains with a mean size of $4.0 \mathrm{~nm}^{2}$, a Y/X growth ratio equal to 2 and an orientation standard deviation of $9^{\circ}$.

In order to demonstrate the ability of the method to gather information on spatial coherence of orientation at both short and long distances we have generated an image corresponding to a loose stacking of concentric circles (concentric cylinders or spheres seen in cross section, that can be considered as rough models of ex-PAN carbon fiber or soot structures), the external circles being $6 \mathrm{~nm}$ in diameter. This image as well as the corresponding local orientation map and the mean orientation difference $(\operatorname{RIMOD}(\rho, \psi))$ are shown in figure 5. The $\operatorname{RIMOD}(\rho, \psi)$ plot shows two main features. First at short distances a quick loss of orientation is observed for $\psi=0^{\circ}$ while the slope is smaller for $\psi=90^{\circ}$, which is due to the perfect stacking of the layer in the radial direction while along the circumference the loss of orientation is due to the curvature of the layers, a maximum disorientation is obtained at $2.6 \mathrm{~nm}$ which corresponds to the mean radius of curvature. Second the $\operatorname{RIMOD}(\rho, \psi)$ plots shows an oscillating behavior at larger distances, this is due to the stacking of the circles, the first minimum is reached at approximately $6 \mathrm{~nm}$ which is the mean distance between two circles.
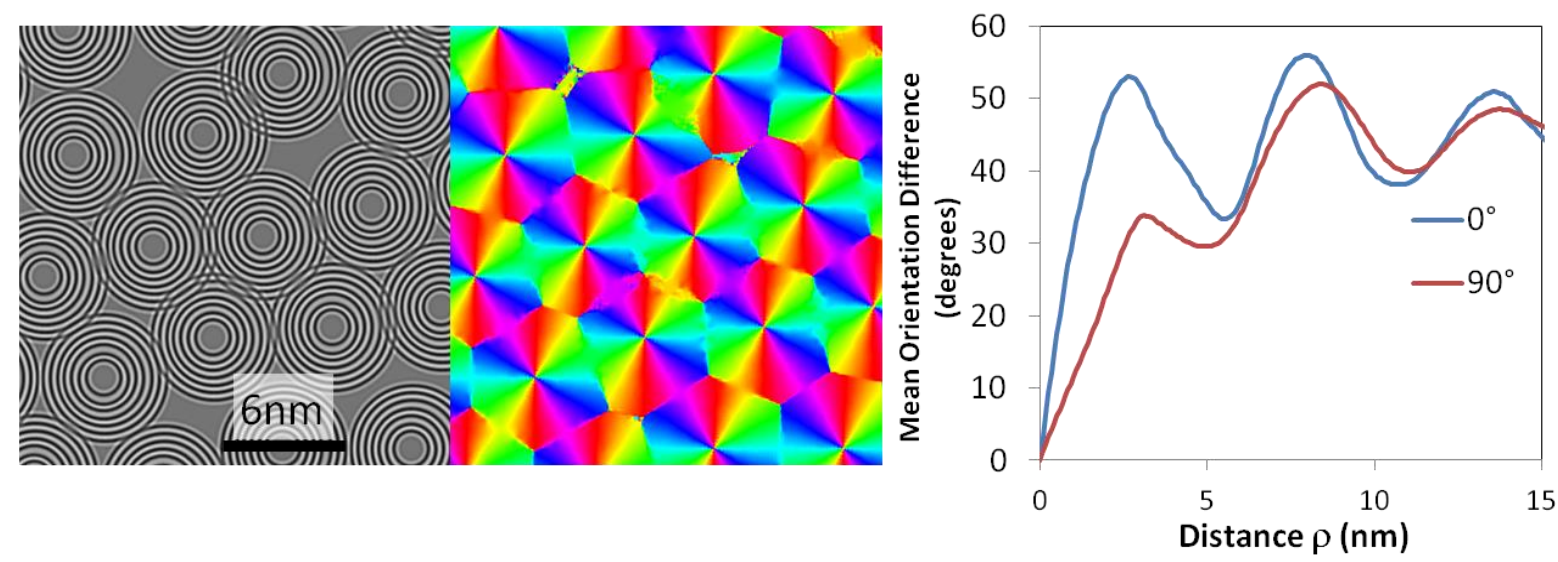

Figure 5: zoom of an artificial image made of a stacking of concentric circles, local orientation and Mean Orientation Difference diagram.

\subsection{Application to lattice fringe images}

In this section, we illustrate how the spatial statistics of orientations can be meaningfully exploited for the characterization of material images. The materials we are interested in are 
four dense carbons observed by high resolution transmission electron microscopy (HRTEM). Four images are represented in figure 6. Resolution is $0.037 \mathrm{~nm} /$ pixel. Image size is $2048 \times 2048$ pixels i.e. about $76 \times 76 \mathrm{~nm}^{2}$ (for clarity reasons, only zooms of $38 \mathrm{~nm}$ and $9 \mathrm{~nm}$ are shown). Depending on the material, the spatial structure of the fringes is more or less regular. For instance, the regenerative laminar pyrocarbon (ReL) and the rough laminar pyrocarbon (RL) are very anisotropic both at low and high scales. Beside their overall anisotropy, a close visual analysis reveals a local organization in stacks of near-parallel fringes. These stacks appear to be larger in RL than in ReL. On the contrary, ex-PAN carbon fiber $\mathrm{C}_{\mathrm{Fiber}}$ and Smooth Laminar PyC (SL) are anisotropic at a very low scale and show much more disorder at higher scales. $\mathrm{C}_{\text {Fiber }}$ fringes are organized in compact wrapped patterns while orientations of fringes in SL images show non stationarities at high scale as indicated in the orientation fields of figure 7.
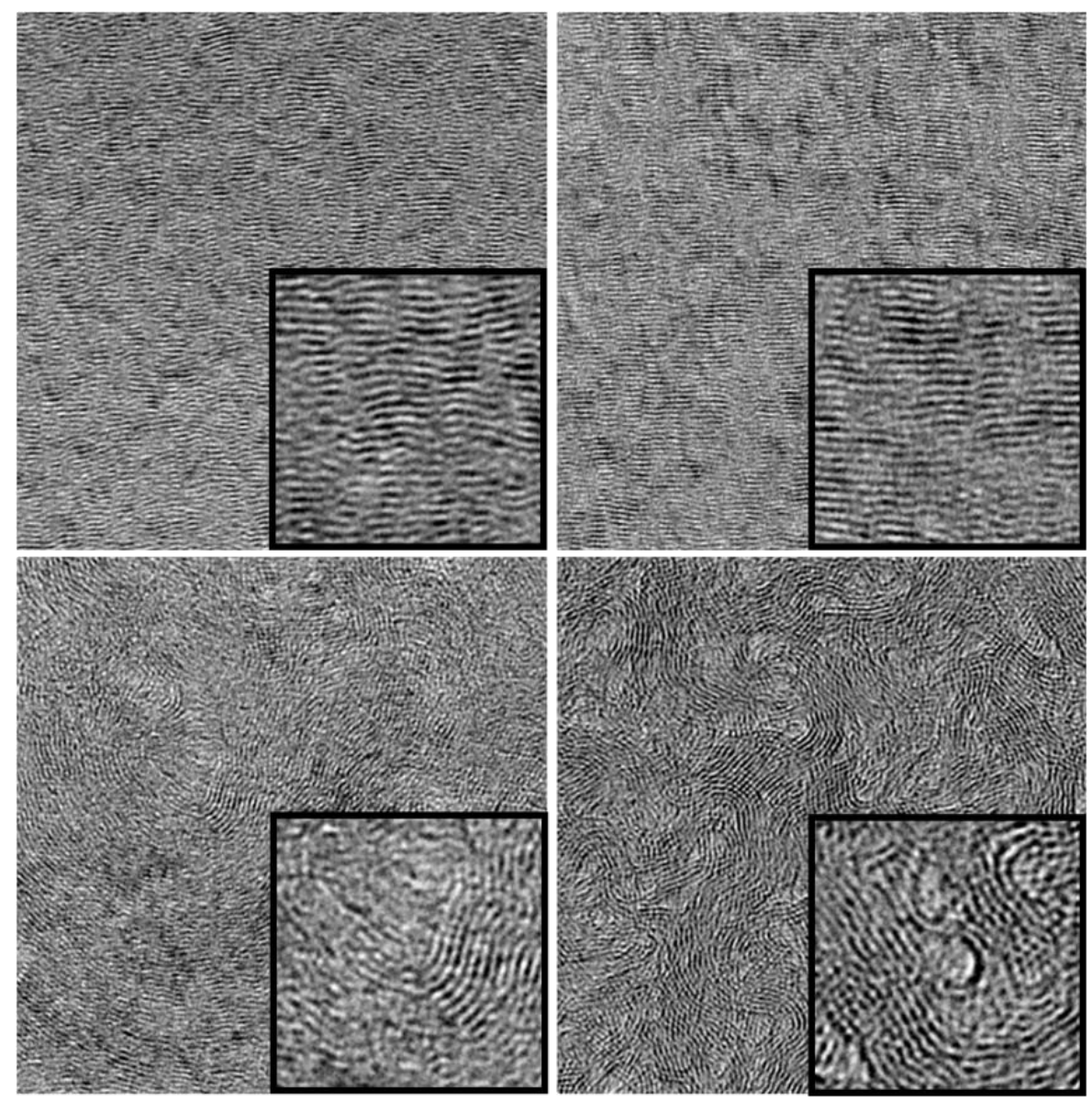

Figure 6: raw HRTEM images of various carbons: upper left ReL, upper right RL, bottom left $\mathrm{SL}$ and bottom right $\mathrm{C}_{\text {Fiber. }}$ Image size is $37.9 \mathrm{~nm} \times 37.9 \mathrm{~nm}$. $9.5 \mathrm{~nm}$ width zooms are shown as inserts. 

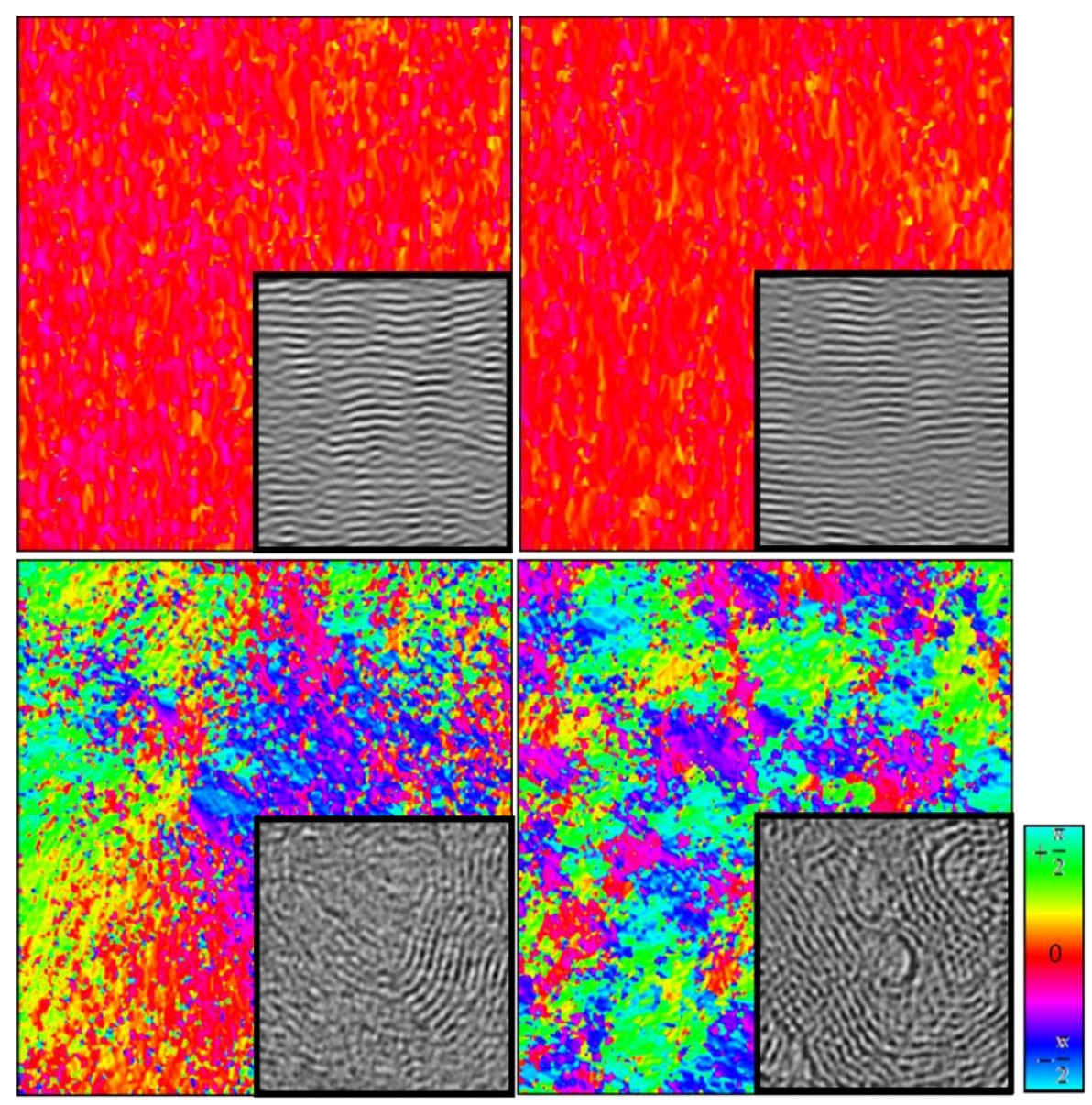

Figure 7: Local orientation for the four carbons (ReL, RL, SL and $\mathrm{C}_{\text {Fiber }}$, from top left to bottom right), orientation palette and zooms of the filtered HRTEM images.

Orientation confidences are shown figure 8 for the four materials, these maps enlighten areas of defects (low confidence) when fringes are too disrupted to allow a good determination of the orientation: dislocations, fringe overlapping, pores, and amorphous areas. The two high textured pyC images exhibit few defects which mainly consist of line defects in the images, few nanometers long, oriented mostly perpendicular to the fringes and located at domains boundaries. The amount of these defective areas is clearly larger for ReL than for RL. SL orientation coherence maps show a high amount of defective areas which are partly due to some fringe overlapping. Indeed, the materials being more isotropic, fringes with different orientations can be found through the thickness of the TEM sample. Note however that fringe overlap has only little effect on the RIMOD statistic as these areas exhibit low confidence values (dark areas in the orientation maps, figure 8) which are used to weight the data. 
Besides, numerous pores are present inside the sample due to the high curvature of the fringes. These pores are also present in $\mathrm{C}_{\mathrm{Fiber}}$ material [30] as well as some fringe overlapping leading to a relatively high amount of defects in the orientation confidence maps. These pores and the disordered areas appear as areas of weak contrast in the HRTEM images.

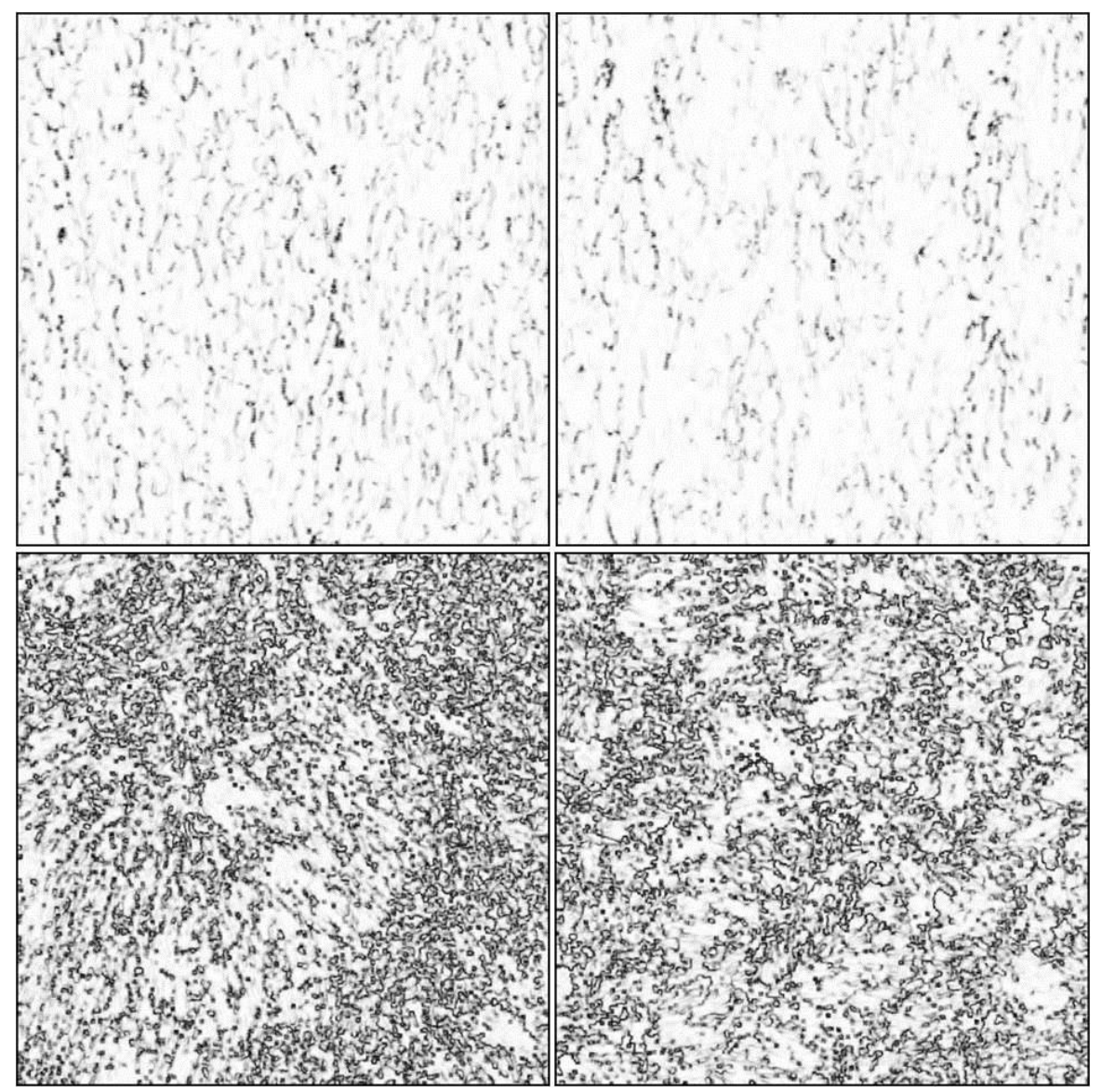

Figure 8: orientation coherences for the four carbons (ReL, RL, SL and $\mathrm{C}_{\text {Fiber }}$, from top left to bottom right).

Orientation fields were obtained on the four images after frequential filtering. Bandpass radial filtering ( $\rho_{0}=2.9 \mathrm{~nm}^{-1}, \alpha=1$ ) was used for the SL PyC and the CFiber; both radial and angular $\left(\alpha=0.5, \sigma_{\theta}=50^{\circ}\right)$ filtering for the two high textured PyCs (RL and ReL) in order to reduce various artifacts such as Bragg fringes or Moirés. It must be pointed out that the image filtering is not mandatory to carry out the analysis and a comparative study of various filters is available as supplementary material. It shows that filtering for high textured materials improves the accuracy of the determination of the orientation at the domain boundaries. When looking at the four orientation fields, it is rather easy to understand that the medium and 
high scale layout of such locally parallel textures is much easier to analyze by studying spatial orientation statistics. In particular, the use of the rotation invariant feature $\operatorname{RIMOD}(\rho, \psi)$ is required here not only because sample orientation changes from an image to another but also because orientation trends change within images due to medium and high scales non stationarities especially in disordered materials such as $\mathrm{C}_{\text {fiber }}$ and SL.

In figure $9, \operatorname{RIMOD}(\rho, \psi)$ is plotted as a function of distance for two angles $\left(\psi=0^{\circ}\right.$ and $\psi=90^{\circ}$ ) and for the four materials. The plots confirm the higher anisotropy of materials ReL and RL compared with SL and $\mathrm{C}_{\text {Fiber }}$. In all cases, orientation spatial differences are low for very short distances and increase to reach an asymptotic trend for long distances corresponding with the twist disorientation $\beta_{M O D}$. This asymptotic trend corresponds to high scale orientation variability. Differences can be observed in the rates of convergence towards the long range variability, the loss of coherence for ReL being faster than for RL. The twist disorientation $\beta_{M O D}$ is also larger for ReL than for $\mathrm{RL}$, respectively $10^{\circ}$ and $8.5^{\circ}$, which is consistent with the orientation angles determined by selected area electron diffraction as shown in table 2. Discrepancies between angles $\psi=0^{\circ}$ and $\psi=90^{\circ}$ are also put into light, revealing that a preferential anisotropy is observed orthogonally to the fringes, especially for material RL.
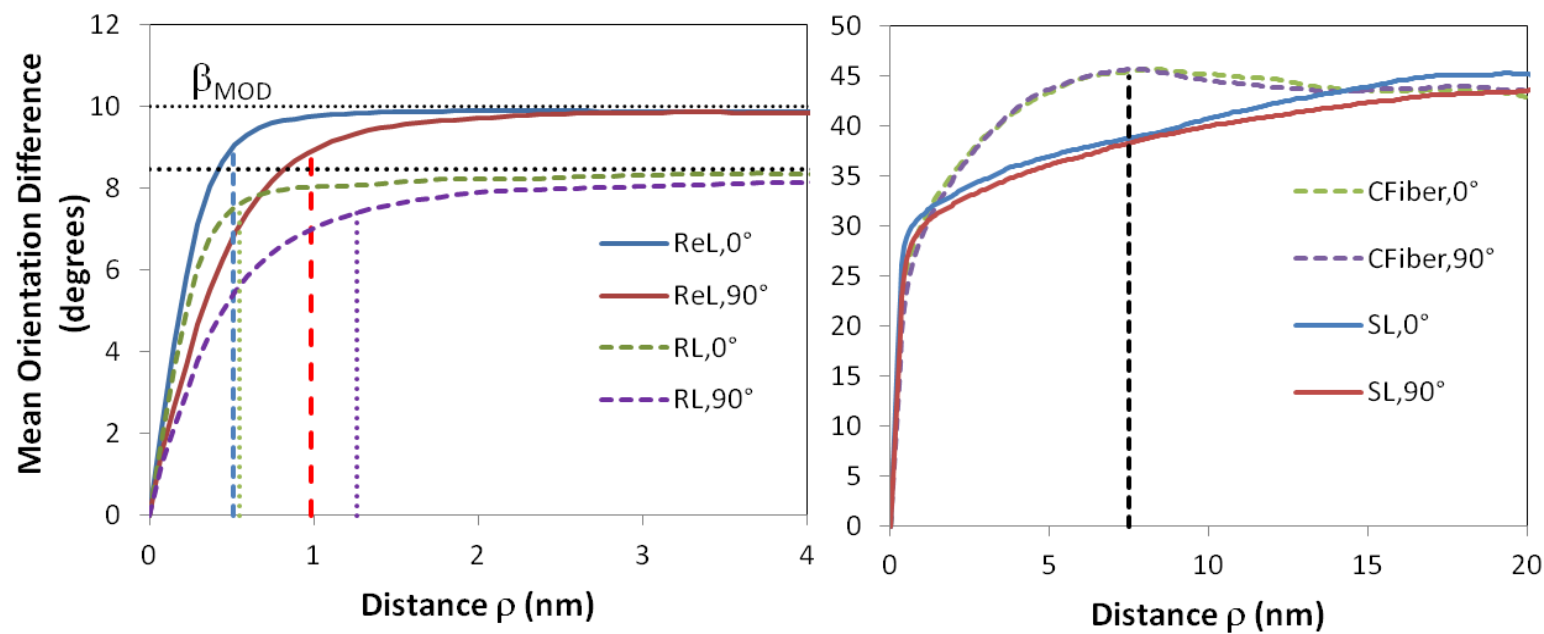

Figure 9: Plot of $\operatorname{RIMOD}(\rho, \psi)$ as a function of distance $\rho$, for angles $\psi=0^{\circ}$ and $90^{\circ}$. Left: ReL and RL PyCs. Vertical dotted lines correspond to the coherence lengths $L_{C M O D}$ and $L_{a M O D}$ and horizontal dotted line to the average disorientation angle $\beta_{M O D}$. Right: SL PyC and $\mathrm{C}_{\mathrm{Fiber}}$, the black dotted line corresponds to the maximum difference of orientation distance for the $\mathrm{C}_{\text {Fiber }}$. 
Coherence lengths $L_{C M O D}$ and $L_{a M O D}$ are determined for both PyCs, $L_{C M O D}=0.99 \mathrm{~nm}$ and $L_{a M O D}=0.51 \mathrm{~nm}$ for ReL and $L_{C M O D}=1.27 \mathrm{~nm}$ and $L_{a M O D}=0.55 \mathrm{~nm}$ for RL. These results show that domains are larger for the RL PyC with a more columnar shape as compared with the ReL PyC. It is of interest to compare these results with the coherence lengths $L_{c}$ and $L_{a}$ determined by X-ray diffraction; results are given table 2 (Standard deviations, given in table 2 have been estimated through the analysis of a set of ten HRTEM images, thus taking into account the spatial variation inside the sample). It appears that coherence lengths obtained by XRD and by MOD are larger for RL than for ReL, however $L_{c}$ and $L_{a}$ obtained by MOD are smaller than the values determined by XRD and the ratio $L_{c} / L_{a}$ is larger for the MOD method than for XRD results. These points will be further discussed below.

\begin{tabular}{|c|c|c|c|c|c|c|}
\hline & $\begin{array}{c}\mathrm{L}_{\text {смоD }}(\mathbf{n m}) \\
\mathbf{\pm 0 . 0 8}\end{array}$ & $\begin{array}{c}\mathrm{L}_{\text {amoD }}(\mathbf{n m}) \\
\mathbf{\pm 0 . 0 2}\end{array}$ & $\begin{array}{c}\mathrm{L}_{\mathrm{c}}(\mathbf{n m})-\text {-XRD } \\
\mathbf{\pm 0 . 1}\end{array}$ & $\begin{array}{c}\mathrm{L}_{\mathbf{a}}(\mathbf{n m})-\text {-XRD } \\
\mathbf{\pm 0 . 3}\end{array}$ & $\begin{array}{c}\boldsymbol{\beta}_{\text {MOD }^{\circ}} \\
\mathbf{\pm 0 . 7}\end{array}$ & $\begin{array}{c}\text { OA }^{\circ} \text { (TEM) } \\
\mathbf{\pm 2}\end{array}$ \\
\hline ReL PyC & 0.99 & 0.51 & 2.9 & 2.4 & 10.0 & 27 \\
\hline RLPyC & 1.27 & 0.55 & 4.1 & 4.2 & 8.5 & 22 \\
\hline
\end{tabular}

Table 2: coherence lengths determined using the Mean Orientation Difference; average orientation angle $\beta_{M O D}$ and coherence lengths determined by XRD for both PyCs. Provided uncertainties for the RIMOD method are the standard deviations estimated over 10 images.

While the curves in figure 9 differentiate between longitudinal and transverse coherence, it is also interesting to figure out how anisotropy varies according to angle $\psi$. This can be done by plotting RIMOD iso-levels using a radar representation. The graphs in figure 10 represent the distance $\rho$ (in nanometers) at which feature RIMOD reaches a value of $\theta$ degrees. $\theta$ is taken in the range $1^{\circ}-9^{\circ}$ for materials $\mathrm{ReL}$ and $\mathrm{RL}$ and in the range $0^{\circ}-35^{\circ}$ for materials $\mathrm{SL}$ and $\mathrm{C}_{\mathrm{Fiber}}$. In the case of ReL and RL, a stronger spatial coherence is observed when $\psi=90^{\circ}$ i.e. orthogonally to the fringes, which suggest that the stacks of near parallel fringes are non isotropic, their height being larger than their width. These iso-level curves also make easier the comparison between samples. For instance, it appears that ReL and RL have similar anisotropy at very short distances whatever the angle $\psi$, but differ at larger distance, as RL seems to have bigger fringe stacks than ReL. 


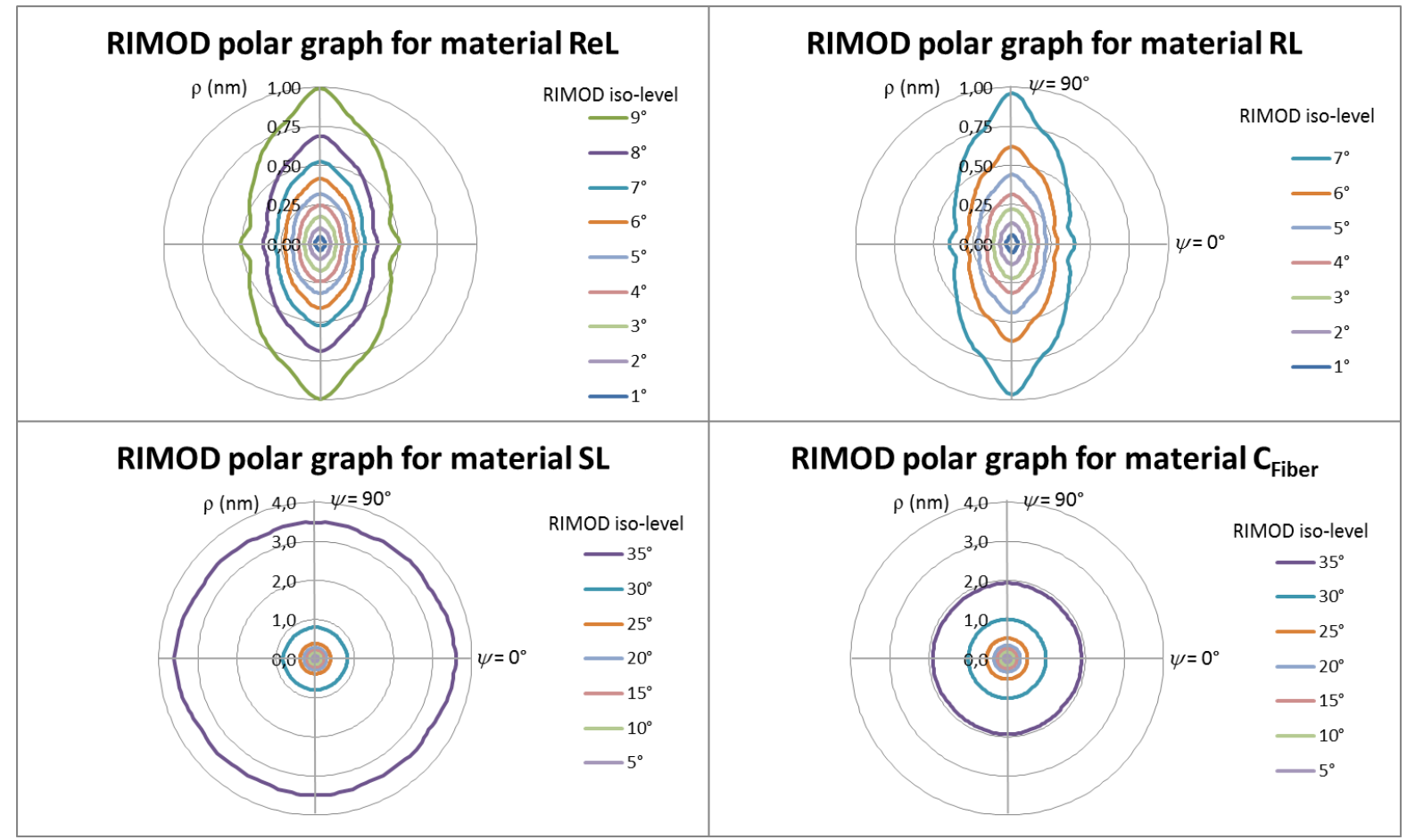

Figure 10: RIMOD iso-level polar graphs computed on the four carbon images (ReL, RL, SL and $\mathrm{C}_{\text {Fiber, }}$, from top left to bottom right). The graphs represent the distance $\rho$ (in nanometers) at which feature $R I M O D$ reaches a value of $\theta$ degrees. $\theta$ is taken in the range $1^{\circ}-9^{\circ}$ for materials $\mathrm{ReL}$ and $\mathrm{RL}$ and in the range $0^{\circ}-35^{\circ}$ for materials SL and $\mathrm{C}_{\mathrm{Fiber}}$.

In order to better understand what quantities are really determined by each method we have performed a more classical analysis of HRTEM images using a fringe detection method based on a level curve tracking algorithm [16]. A zoom of the HRTEM image of RL and the corresponding fringes detected are shown fig. 11.
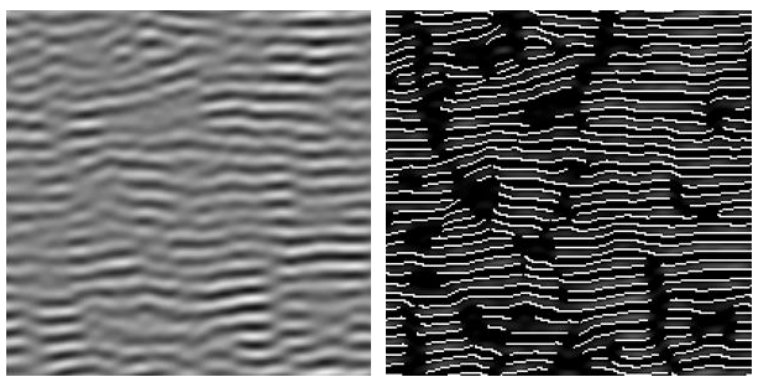

Figure 11: zoom of an HRTEM image for the ReL PyC (left) and detected fringes (right). 
This procedure allows obtaining a distribution of fringe sizes, which are shown at figure 12 for ReL and RL. It appears that the coherence length $L_{a M O D}$ is close to the mode of the distribution of fringe lengths while by XRD $L_{a}$ values are shifted towards the largest fringe lengths. Two reasons could explain the larger values obtained by XRD. First, the CarbonXS program assumes a Gaussian distribution of stacking sizes whereas real domain size distributions are usually log-normal, leading to an unrealistic mean value. Second, the coherence lengths determined by full profile matching XRD methods [34-35] are length weighted values (second moment the distribution divided by the first moment) [35], which are usually greater than the arithmetic mean; it can be seen in figure 2 that the length weighted average for the ReL PyC $\left(L_{L W}-R e L\right)$ is similar to $\mathrm{L}_{\mathrm{a}}(\mathrm{XRD})$ while the one for the RL PyC is slightly smaller than $L_{a}(3 \mathrm{~nm}$ from the fringe length distribution and $4.1 \mathrm{~nm}$ from XRD). Furthermore it must be underlined that XRD results are averaged over a large volume of material while TEM results were obtained on a much small amount and are thus less statistically significant.

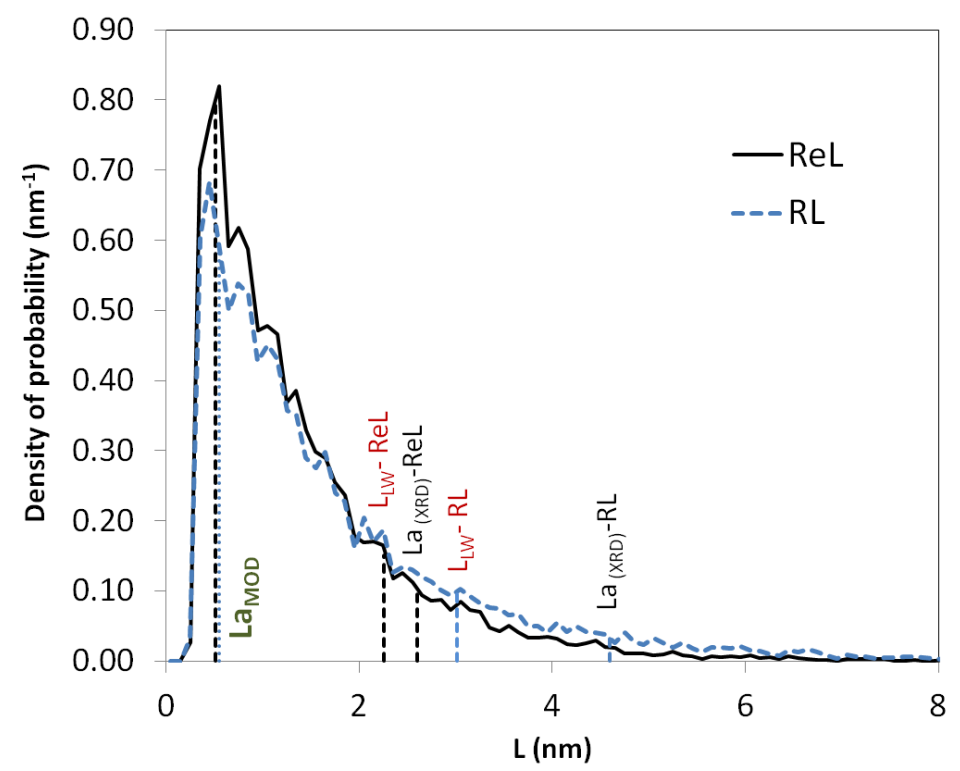

Figure 12: Fringe length distribution determined for ReL (continuous line) and RL (dotted line). Abscissae corresponding to $L_{a M O D}, L_{a(\mathrm{XRD})}$ are reported on the diagram as well as the length-weighted averages $\left(\mathrm{L}_{\mathrm{LW}}\right)$, calculated from the fringe length distributions.

Moreover the structural complexity of turbostratic carbons makes their nanostructure deviate far from the schematic representation of the BSU (see figure 13) commonly accepted to describe the structural parameters determined by HRTEM and XRD. 


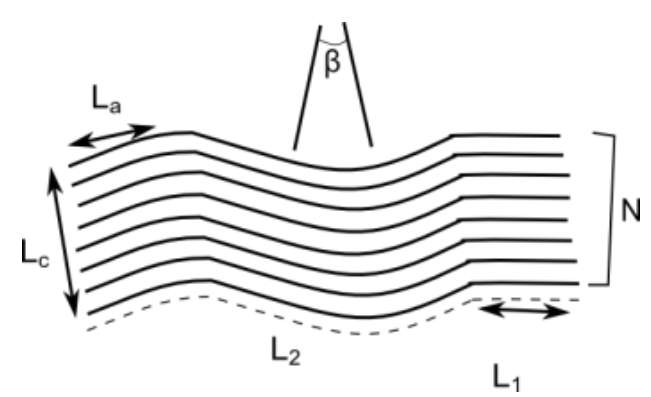

Figure 13: Representation of a basic structural unit of a turbostratic carbon, according to [52], $L_{1}, L_{2}$ and $\beta$ (twist disorientation angle) obtained by high resolution transmission electron microscopy. $L_{c}$ (average stack height) and $L_{a}$ (average graphene layer extent) are determined by X-ray diffraction and are length-weighted averages.
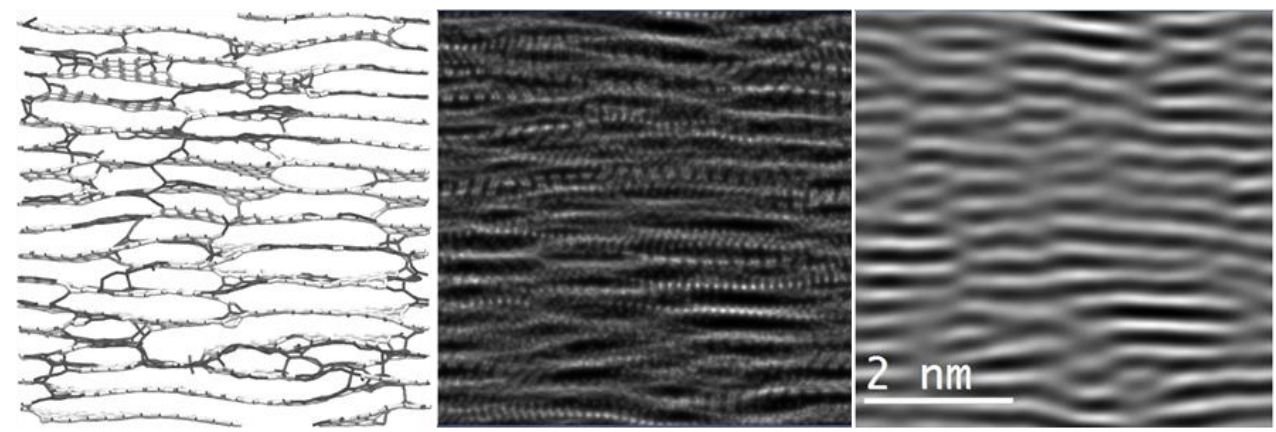

Figure 14: From left to right: slice of a model of pyrocarbon according to Leyssale et al. [8], projected potential and corresponding simulated HRTEM image.

Indeed, it is only recently that a realistic representation of the atomistic structure of some pyrolytic carbons has been proposed [8]. A slice of such a model is shown figure 14 as well as the projected electrostatic potential and the corresponding simulated HRTEM image, PyCs are made of wrinkled aromatic layers containing 5 and 7 membered rings; these layers are turbostratically stacked and bonded together by screw dislocations and $\mathrm{sp}^{3}$ atoms. The resulting lattice fringe image is similar to a projection of the structure along the viewing direction and contains more or less contrasted fringes according to their orientation (tilt angle), their extension and overlap through the foil thickness and the presence of defects. Thus the structural parameters $L_{a M O D}$ and $L_{c M O D}$ give an information averaged over the perfectly ordered and the defective areas (which are however weighted by the confidence factor) and are consequently smaller than the average fringe lengths. Besides, the large difference between the $L_{C M O D} / L_{C M O D}(\sim 2)$ ratio and the $L_{c(X R D)} / L_{a(X R D)}$ ratio $(\sim 1)$ could be due to the projection effect. Indeed, the thin slice thickness is estimated to be in the range 10-30 
$\mathrm{nm}$ and thus a large number of domains are superimposed along the thickness. Larger models $(>10 \mathrm{~nm})$ with known domain sizes will be needed to confirm this hypothesis.

Concerning materials $\mathrm{C}_{\mathrm{Fiber}}$ and $\mathrm{SL}$, the rates of convergence are especially noteworthy. From figure 9 it can be observed that at short distances, the loss of coherence is slightly faster for SL (below $\rho=1 \mathrm{~nm}$ ) but slows down afterwards and becomes slower than for material $\mathrm{C}_{\text {Fiber }}$ that reaches its largest value $\left(\operatorname{RIMOD}(\rho, \psi)=45.6^{\circ}\right)$ for $\rho=7.5 \mathrm{~nm}$, at a continuous rate; then, more surprisingly, for $\mathrm{C}_{\mathrm{Fiber}}, R I M O D$ slightly decreases for larger distances $\left(\operatorname{RIMOD}(\rho, \psi)=43.6^{\circ}\right.$ for $\left.\rho=15 \mathrm{~nm}\right)$. This behavior can be explained by the specific microstructure of ex-PAN carbon fibers; they are made of a deep entanglement of folded sheets of layers, crumpled along the fiber axis, a microstructure fairly similar to the stacking of concentric cylinders artificially generated fig. 5. The curvature of the sheets are rather homogeneous as can be seen on the HRTEM image, fig. 6, which results in basic structural units with a mean curvature radius of roughly $8 \mathrm{~nm}$, clearly visible in the local orientation map (fig. 7). The slight decrease of RIMOD at larger distances may be due to the parallelism between neighboring BSUs which have a ribbon-like shape; however the oscillating feature shown in figure 5 is not observed in practice probably because the domains are not perfectly cylindrical and have various curvatures and sizes.

\section{Conclusion}

A new method of analysis of the HRTEM images of turbostratic carbons such as pyrocarbons or carbon fibers has been proposed. Contrary to standard procedures this method does not detect individual fringes but finds their local orientation and look at the spatial correlation between orientations. An analysis based on second-order statistics of the orientation field allows achieving a quantitative description of the nanostructure: coherence lengths $L_{a M O D}$ and $L_{C M O D}$ give the mean dimension of the BSU parallel and perpendicular to the fringes and $\beta_{M O D}$ is a determination of the mean disorientation of fringes. A more thorough analysis of the mean orientation difference plots can give information such as the curvature of the USB and the correlation of orientations at large distance.

The method has been applied to the characterization of two high textured pyrocarbons. The results have been compared with some experimental data obtained by X-ray diffraction and electron diffraction. All techniques give consistent results and show that the rough laminar PyC exhibits larger coherent domains and is more textured at every length scale than the regenerative laminar PyC. Moreover the orientation field analysis provides a clear 
visualization of the defects, evidenced in the HRTEM images of high textured PyCs as line defects, perpendicular to the fringes and located at the domain boundaries. This method has already been used to validate some atomistic models of pyrocarbons [9] and thus can help in the generation of more realistic models.

The application of the method on two less textured carbons (smooth laminar PyC and ex-PAN carbon fiber) showed that it can reveal information at different length scales: at short lengths scale $(<2 \mathrm{~nm})$ the extent of domain sizes are revealed, while at larger length scale $(>4 \mathrm{~nm})$ information such as the heterogeneity of the sample (SL PyC) or the presence of regularly spaced tubular structures $\left(\mathrm{C}_{\text {Fiber }}\right)$ are enlightened.

Future works will focus on two points: first, on a better recognition of the amorphous areas, holes and images of lattices planes perpendicular to the electron beam in order to extend the method to more complex images such as HRTEM images of soot or activated carbons and second, on the determination of domain sizes $\left(L_{a M O D}, L_{C M O D}\right)$ on these less organized carbons.

\section{Acknowledgements}

Funding from the French Agence Nationale de la Recherche through the PyroMaN project (contract ANR-2010-BLAN-929) and from the Carnot Materials and systems Institute of Bordeaux (MIB) are gratefully acknowledged. The authors are grateful to Professor Jeff Dahn for kindly providing the CarbonXS program.

\section{References}

[1] Fernandez-Alós V, Watson JK, Vander Wal RL, Mathews JP. Soot and char molecular representations generated directly from HRTEM lattice fringe images using Fringe3D. Combust \& Flame 2011; 158(9):1807-13.

[2] Pré P, Huchet G, Jeulin D, Rouzaud JN, Sennour M, Thorel A. A new approach to characterize the nanostructure of activated carbons from mathematical morphology applied to high resolution transmission electron microscopy images. Carbon 2013; 52:239-58.

[3] Shim H-S, Hurt RH, Yang NYC. A methodology for analysis of 002 lattice fringe images and its application to combustion-derived carbons. Carbon 2000; 38(1):29-45.

[4] Mathews JP, Van Duin ACT, Chaffee AL. The utility of coal molecular models. Fuel Process Technol 2011; 92(4):718-28. 
[5] Vander Wal RL, Tomasek AJ, Pamphlet MI, Taylor CD, Thompson WK. Analysis of HRTEM images for carbon nanostructure quantification. J Nanopart Res. 2004; 6:55568.

[6] Reznik B, Hüttinger KJ. On the terminology for pyrolytic carbon. Carbon 2002; 40(4):617-36.

[7] Bourrat X, Fillion A, Naslain R, Chollon G, Brendlé M. Regenerative laminar pyrocarbon. Carbon 2002; 40(15):2931-45.

[8] Leyssale J-M, Da Costa J-P, Germain C, Weisbecker P, Vignoles GL. Structural features of pyrocarbon atomistic models constructed from transmission electron microscopy images. Carbon 2012; 50(12):4388-400.

[9] Farbos B, Weisbecker P, Fischer HE, Da Costa JP, Lalanne M, Chollon G, Germain C, Vignoles GL, Leyssale J-M. Nanoscale structure and texture of highly anisotropic pyrocarbons revisited with transmission electron microscopy, image processing, neutron diffraction and atomistic modelling. Carbon 2014; 80:472-89.

[10] Ammar MR, Rouzaud J-N, Vaudey CE, Toulhoat N, Moncoffre N. Characterization of graphite implanted with chlorine ions using combined Raman microspectrometry and transmission electron microscopy on thin sections prepared by focused ion beam. Carbon 2010; 48(4):1244-51.

[11] Gotoh Y, Shimizu H, Murakami H. High resolution electron microscopy of graphite defect structures after keV hydrogen ion bombardment. J Nucl Mater 1989; 162-164(C): 851-855.

[12] Sharma A, Kyotani T, Tomita A. A new quantitative approach for microstructural analysis of coal char using HRTEM images. Fuel 1999; 78(10):1203-12.

[13] Rouzaud J-N, Clinard C. Quantitative high-resolution transmission electron microscopy: a promising tool for carbon materials characterization. Fuel Processing Technology 2002; 77:229-35.

[14] Yehliu K, Vander Wal RL, Boehman AL, Development of an HRTEM image analysis method to quantify carbon nanostructure. Combust \& Flame 2011; 158(9):1837-51.

[15] Tóth P, Palotás AB, Lighty J, Echavarria CA. Quantitative differentiation of poorly ordered soot nanostructures: a semi-empirical approach. Fuel 2012; 99:1-8.

[16] Da Costa J-P, Germain C, Baylou P. Level Curve Tracking Algorithm for Textural Feature Extraction. Proc. 15 ${ }^{\text {th }}$ Intl. Conf. on Pattern Recognition, Barcelona, Spain, 2000; 3:909-12. 
[17] Da Costa J-P, Germain C, Baylou P. A Curvilinear Approach for Textural Feature Extraction: Application to the Characterization of Composite Material Images. In: Procs. Intl. Conf. on Quality Control by Artificial Vision, Le Creusot, France, 2001. 2001:Cépaduès, Toulouse, France.

[18] Raynal P-I, Monthioux M, Da Costa J-P, Dugne O. Multi-scale quantitative analysis of carbon structure and texture: III. Lattice fringe imaging analysis. Procs. Carbon 2010 Conference, Clemson, SC, USA, 11-16 July 2010, M. Thies ed.; 2010, ref. 627.

[19] Oberlin A. High-resolution TEM studies of carbonization and graphitization. Chem Phys Carbon 1989; 22:1-143.

[20] Rouzaud J-N, Oberlin A. Structure, microtexture, and optical properties of anthracene and saccharose-based carbons. Carbon 1989; 27(4):517-29.

[21] Tóth P, Farrer JK, Palotás AB, Lighty JS, Eddings EG. Automated analysis of heterogeneous carbon nanostructures by high-resolution electron microscopy and online image processing. Ultramicroscopy 2013; 129:53-62.

[22] Tóth P, Palotás AB, Eddings EG, Whitaker RT, Lighty JA. A novel framework for the quantitative analysis of high resolution transmission electron micrographs of soot $\mathrm{I}$. Improved measurement of interlayer spacing. Combust \& Flame 2013; 160(5):909-19.

[23] Tóth P, Palotás AB, Eddings EG, Whitaker RT, Lighty JA. A novel framework for the quantitative analysis of high resolution transmission electron micrographs of soot II. Robust multiscale nanostructure quantification. Combust \& Flame 2013; 160(5):92032.

[24] Freeman TW, Adelson EH. The design and use of steerable filters. IEEE Trans. Pattern Anal. \& Machine Intellig.1991; 13(9):906-81.

[25] Germain C, Da Costa J-P, Lavialle O, Baylou P. Multiscale estimation of vector field anisotropy - application to texture characterization. Signal Processing 2003; 83(7):1487-503.

[26] Oberlin A. Pyrocarbons. Carbon 2002; 40(1):7-24.

[27] Goma J, Oberlin A. Characterization of low temperature pyrocarbons obtained by densification of porous substrates. Carbon 1986; 24 (2):135-42.

[28] Weisbecker P, Leyssale J-M, Fischer HE, Honkimäki V, Lalanne M, Vignoles GL. Microstructure of pyrocarbons from pair distribution function analysis using neutron diffraction. Carbon 2012; 50(4):1563-73.

[29] Weisbecker P, Lalanne M, Farbos B, Chollon G, Leyssale J-M, Vignoles GL, Fischer HE. A Pair Distribution Function approach to pyrocarbon structure using neutron 
diffraction. Carbon 2012 Conference, Kraków, Poland; S. Blazewicz \& E. Frackowiak eds.; ref. 430.

[30] Guigon M, Oberlin A, Desarmot G. Microtexture and Structure of Some High-Modulus, PAN-Based Carbon Fibres. Fibre Sci. \& Technol. 1984; 20:177-98.

[31] Weisbecker PW, Guette A. Thin-film preparation of C/C composites and CMC using the broad argon ion beam method. Procs $17^{\text {th }}$ Intl. Conf. on Composite Materials, Edinburgh, UK, 2009. Available online http://www.iccm-central.org/Proceedings/ ICCM17proceedings/Themes/Materials/.

[32] Tanabe T. Radiation damage of graphite - Degradation of material parameters and defect structures. Physica Scripta T 1996; 64:7-16.

[33] Shi H, Reimers JN, Dahn JR. Structure-refinement program for disordered carbons. J Appl Crystallogr 1993; 26:827-36.

[34] Ruland W, Smarsly B. X-ray scattering of non-graphitic carbon: An improved method of evaluation. J Appl Crystallogr 2002; 35(5):624-33.

[35] Faber K, Badaczewski F, Ruland W, Smarsly BM. Investigation of the microstructure of disordered, non--graphitic carbons by an advanced analysis method for wide-angle Xray scattering. Zeitschr Anorg u Allg Chemie 2014; DOI:10.1002/zaac.201400210

[36] Haralick R. Statistical and structural approaches to textures. Procs IEEE 1979; 67(5):786-804.

[37] Unser M. Sum and difference histograms for texture classification. IEEE Trans. on Pattern Anal \& Machine Intellig 1986; 8(1):118-25.

[38] Chetverikov D. Texture analysis using feature-based interaction maps. Pattern Recognition 1999; 32(3):487-502.

[39] Davis L, Johns SA, Aggarwal JK. Texture analysis using generalized co-occurrence matrices. IEEE Trans. on Pattern Anal \& Machine Intellig 1979; 1(3):251-9.

[40] Kovalev V, Petrou M. Multidimensional co-occurence matrices for object recognition and matching. Graphical Models \& Image Process. 1996; 58(3):187-97.

[41] Kovalev V, Petrou M, Bondar Y. Using orientation tokens for object recognition. Pattern Recogn Lett 1998; 19(12):1125-32.

[42] Kass M, Witkin A. Analysing oriented patterns. Computer Vision, Graphics \& Image Processing 1987; 37(3):362-85.

[43] de Luis-Garcia R, Deriche R, Alberola-López C. Texture and color segmentation based on the combined use of the structure tensor and the image components. Signal Processing 2007; 88(4):776-95. 
[44] Bigün J, Granlund G, Wiklund J. Multidimensional orientation estimation with applications to texture analysis and optical flow. IEEE Trans. Pattern Anal. \& Machine Intellig. 1991; 13 (8):775-89.

[45] Le Pouliquen F, Da Costa J-P, Germain C, Baylou P. A new adaptive framework for unbiased orientation estimation. Pattern Recognition 2005; 38(11):2032-46.

[46] Michelet F, Da Costa J-P, Lavialle O, Berthoumieu Y, Baylou P, Germain C. Estimating local multiple orientations. Signal Processing 2007; 87(7):1655-69.

[47] Leyssale J-M, Da Costa J-P, Germain C, Weisbecker P, Vignoles GL. An image guided atomistic reconstruction of pyrolytic carbons. Appl Phys Lett 2009; 95(23):231912.

[48] Krieger G, Zetzsche C. Nonlinear image operators for the evaluation of local intrinsic dimensionality. IEEE Trans Image Process. 1999; 5(6):1026-42.

[49] Mardia K, Jupp J. Directional Statistics. John Wiley \&Sons, Chichester, England; 2000.

[50] Da Costa J-P, Germain C, Baylou P. Orientation difference statistics for texture description. In: Procs. $16^{\text {th }}$ Intl. Conf. on Pattern Recognition, Québec, Canada, 2002; $1: 652-5$.

[51] Chetverikov D, Haralick R. Texture anisotropy, symmetry, regularity: recovering structure and orientation from interaction maps. Procs. $6^{\text {th }}$ British Machine Vision Conference, Birmingham, UK, 1995; 57-66.

[52] Bhushan B. Springer handbook of nanotechnology. $1^{\text {st }}$ ed.; 2004: Springer, Berlin. 